\title{
An Automatic Identification Algorithm of Forest Fire Based on Visible Light
}

\author{
Aijun $\mathrm{Xu}^{1,2}$ Luming Fang ${ }^{2}$ Zile Tang ${ }^{2}$ \\ ${ }^{1}$ National Laboratory for Information Engineering in Surveying, Mapping and Remote Sensing, Wuhan University, \\ Wuhan, 430079, P.R.China \\ ${ }^{2}$ School of Information Engineering, Zhejiang Forestry University, Lin'an, 311300, P.R. China
}

\begin{abstract}
In RGB space, the paper optimizes the algorithm of median filter. Not only it could keep its original filter capability, but also it makes the quantity of algorithm decrease greatly. The paper puts forward a standard for identifing fire based on the fire edge wobblingsharp features standard. There are two key problems in the sharp features standard: identification of sharp features and how to identify the threshold of sharp wobbling. And the paper evaluates the algorithm of fire identification with the recall ratio and the precision ratio.
\end{abstract}

Keywords: Visible light, Forest fire, Fire identification, Median filter

\section{Introduction}

The fire automatic identification technology based on visible light is widely used. This technology makes the video's processing and management go deep into the media level, which enable users to handle and process different kinds of video information. Identifying and managing the flame luminance of furnace in early time, and establishing flame image characters database are some experimentation based on automatic fire identification. Nowadays this technology has developed and be used in more applications, such as forest fire prevention, indoor fire alarm, architecture fire prevention system, fire simulation system, machinery manufacture and entertainment education[1]-[13]. In boiler surveillance system, analyzing several exits' flame of video surveillance and digging out the flame's temperature and burning mode, can provide desicion supports for controlling the fire of boiler. In the region of forest fire prevention, identifying forest fire image by making use of fire identification based on visible light can help forestry management department discover forest fire disaster in time[13].

This paper mainly researches on the technology of forest fire identification based on visible light.The research object in this paper is the video monitoring data, and to discuss forest fire identification based on visible light with long distance real-time delivering of images. According to the background, the target and the moving object of the real-time video data and the color, texture, shape in image data, the algorithm may find out the images which can satisfy special visual characteristic in the existed characteristic database.

\section{Image pre-processing}

\subsection{Methods of image pre- processing}

The images obtained by CCD pickup camera can't be used in identifying fire directly, and they should be pre-processed. The meaning of image pre-processing is to transform the original images into what computer can identify, and to make them conform to the demands of computer vision[3]-[5]. Image preprocessing includes some steps as follows: median filter of RGB data, median filter of the binaryzation data, Identifying color characteristics, filtering isolated point, deleting noisy point, etc[10]-[12].

Median filter of RGB data is convenient for the computer to process images. Nowadays, the RGB model is the most common color model for the hardware[6], [7].

According to the achievements from many scholar, the value range of flame parameter in the color space of RGB is as follows:

$$
(R, G, B)=\left\{\begin{array}{l}
117 \leq R \leq 255 \\
88 \leq G \leq 255 \\
44 \leq B \leq 255
\end{array}\right.
$$

With median filter of RGB data, it can not only smooth the noise area, but also keep the edge constructure among image areas, and it could initially delete some noise points.

Median filter of the binaryzation data makes the edge tend to be obvious, and it is good for distinguishing the foreground and the background of image, Furthermore, it further deletes some isolated core color points. 


\subsection{The algorithm of median filter and its improvement}

Median filter is a very useful non-liner method in processing images, and its basic principle is to design a moving window, and make the window move in the images, then to sort all gray value of pixels in pixel region which is covered by the moving window, and substitute its median values for the original gray value in the window central pixel.

In RGB space, the paper optimizes the algorithm of median filter. Not only it could keep its original filter capability, but also it makes the quantity of algorithm decrease greatly. Its basic thought is to fuse liner median filter and non-liner vector median filter. It carries on traditional median filter, then uses the liner average operation, and the artificial way is a complement to compute the liner average at last.The algorithm's thought is as follows:

Every image can be expressed in the form of $F=[f(i, j)]$, and $(i, j)$ represents pixel's coordinate, $f(i, j)$ represents the gray value of pixels, the $F$ represents the assembly of all pixels in the form of matrix. $W[f(i, j)]$ represents window processing with the pixels in the images, and $g(x, y)=\operatorname{median}(\mathrm{W}[\mathrm{f}(\mathrm{i}, \mathrm{j})])$ represents calculate median values from the pixels in the window of $W[f(i, j)]$.

There the paper takes the window of $3 \times 3$ as an example to illustrate the principle of median filter after being optimized. The calculating method is as follows:

$$
F=\left[\begin{array}{lcc}
(x-1, y+1) & (x, y+1) & (x+1, y+1) \\
(x-1, y) & (x, y) & (x+1, y) \\
(x-1, y-1) & (x, y-1) & (x+1, y-1)
\end{array}\right]
$$

(1) To put $y=y^{\prime} *$ width into the matrix F. (width represents the width of images.)

(2) To calculate the gray value $a_{i j}$ separately, $a_{i j}$ represents the element in row $i$ and column $j$, then it can get a new matrix A:

$$
A=\left[\begin{array}{lll}
a_{11} & a_{12} & a_{13} \\
a_{21} & a_{22} & a_{23} \\
a_{31} & a_{32} & a_{33}
\end{array}\right]
$$

(3) To calculate average by the formula $\overline{f(x, y)}=\frac{1}{M \times N} \sum_{i=1}^{3} \sum_{j=1}^{3} a_{i j}, M \times N$ represents the size of matrix, at this time $M \times N=3 \times 3=9$.

(4) considering the error may be occured in mathematical operation, in order to reduce the quantity of algorithm and save time, th paper puts forward an correction factor $\lambda$ which is used to correct $g(x, y)$. $\lambda$ is a experience value, and after several tests we made $\lambda=0.5$.
(5) According to the math expression $g(x, y)=\overline{f(x, y)}+0.5$, we can carry on calculating median value based on correction immediately.

\section{Algorithm of fire identification based on visible light}

\subsection{Algorithm thought}

The fire flame in early time is unstable and developmental constantly. The forest fire remote video surveillance system just could identify the special shape and radiation characteristics of the fire flame in early time[9]. The unstable flame has many sharp features, and there is an obvious phenomenon when the fire edge is wobble: the number of the flame's sharp features appears an irregular change $[8,10]$. So the paper puts forward a standard to identify fire based on the fire edge wobbling ----sharp features standard. There are two key problems in the sharp features standard: identification of sharp features and how to identify the threshold of sharp wobbling, that also means to find out the difference in sharp wobbling between the fire flame in early time and other luminous.

The algorithm's main design thought is: firstly, obtains two images in video flow at regular intervals, finds out the common area, and takes the common area as the third image; secondly, obtains two images in video flow at regular intervals again, finds out the common area, and takes the common area as the sixth image; finally, and to find out the common area between the third image and the sixth image, and judges whether the common area is fire area. Its algorithm flow is as figure 1:

(1) According to the methods of image preprocessing in the chapter 2, obtain the first eligible binaryzation image pic1;

(2) Using a timer to obtain and deal with the second picture named pic2 at regular intervals;

(3) Carrying on matching operation for the pic 1 and pic2 to get common area, if the proportion of the common area in the whole area is bigger than a threshold, then it is named pic3;

(4) Executing the first step to the third step repeatedly, to obtain pic4, pic5 and the common area between them in turn. if the proportion of the common area in the whole area is bigger than a threshold, then it is named pic6;

(5) Obtaining a common region from pic3 and pic6, then this common area is named as pic7. if the proportion of the common area in the whole area is bigger than a threshold, than it maked a sound alarm. 


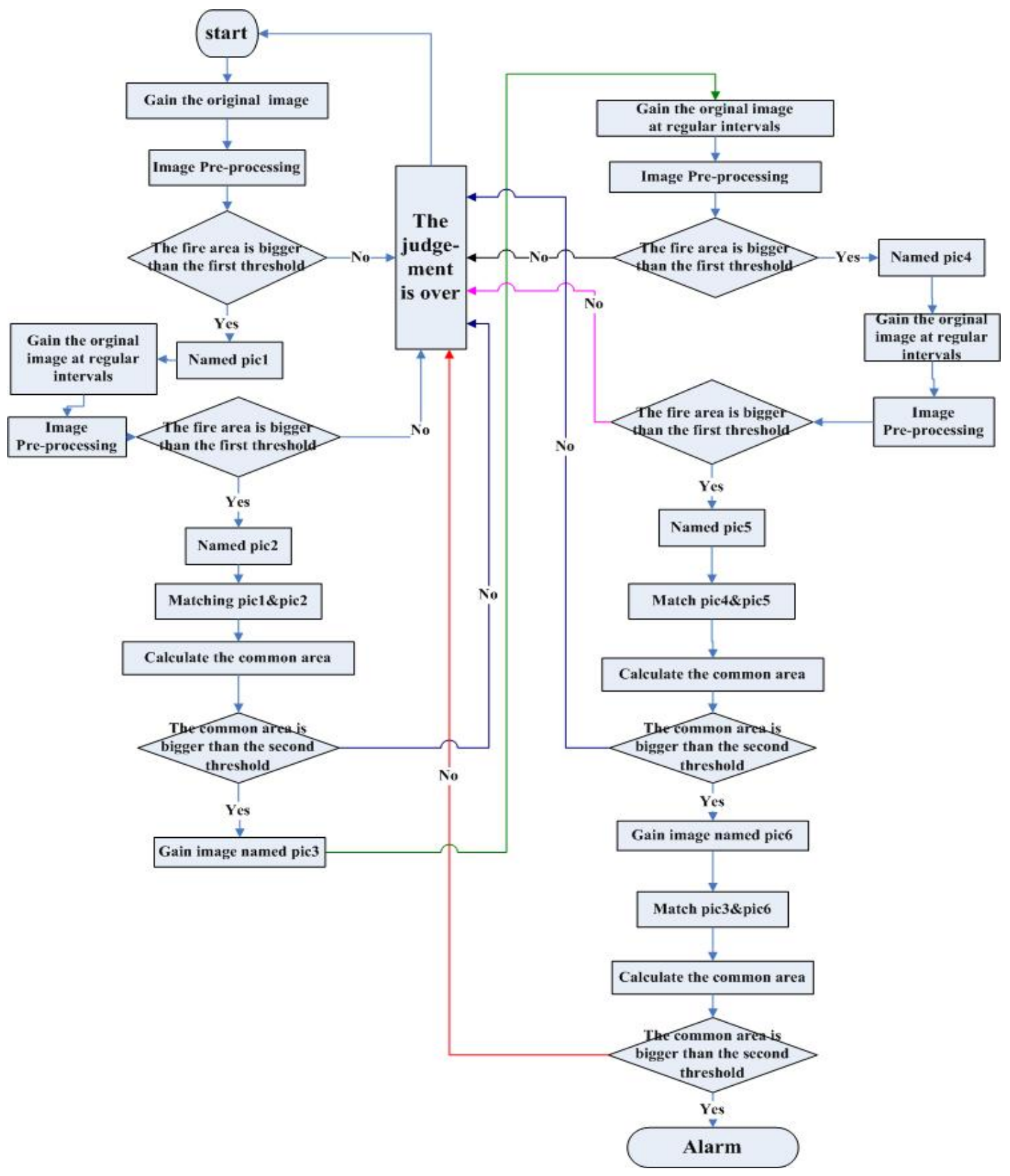

Fig.1: flow of the algorithm

\subsection{The judgment of fire area}

In order to obtain the differences of color feature values in image from flame area and background area in different environments, and the differences of color feature between flame and other luminous object, such as vehicle lights, flashlight and so on. The authors build the flame color feature database for forest fire based on many experiments and experience data, the database is as follows:

$$
R G B(r, g, b)=\left\{\begin{array}{l}
r=255 \\
183 \leq g \leq 255 \\
15 \leq b \leq 219
\end{array},\left\{\begin{array}{l}
250 \leq r \leq 254 \\
g=255 \\
15 \leq b \leq 219
\end{array}\right\}\right.
$$

The implementation is as follows:

Getting a image $I_{A}$ and matching color feature of every pixel in the image with the RGB value in the flame color feature database, the processing steps are as follows:

(1) getting a color value of a point $(x, y)$ which has not been processed. 
(2) decomposing the color into three components of R, G, B.

According to the mathematical expression: $\mathrm{p}=\mathrm{x} * 3+\mathrm{y} * \mathrm{~m}$ nWidth*3, it can get the formula as follows ( $\mathrm{m}$ nWidth represents the width of the image).

$$
\left\{\begin{array}{l}
r=\mathrm{pRGB}[\mathrm{p}] \\
\mathrm{g}=\mathrm{pRGB}[\mathrm{p}+1] \\
\mathrm{b}=\mathrm{pRGB}[\mathrm{p}+2]
\end{array}\right.
$$

In the formula, $p R G B$ represents converting $\mathrm{p}$ into an binary unsigned integer of 8 bits and being showed out in the form of decimal system. Then the color value of the central part in this point is $R G B(r, g, b)$.

(3) connecting the flame color feature database, and carrying on the similarity examination between the color value of the point and the database.

If the similarity is high, then it means that the color of this point is the flame's core color.

If the similarity is low, then the point is treated as a noisy point.

(4) processing every pixel in the image according to the above steps.

\section{The algorithm performance analysis}

The main indexes which can evaluate the algorithm of fire identification are the recall ratio and the precision ratio.

The precision ratio is the ratio of the identified images (which really have flame) and all retrieval images (which really have flame and have not flame but algorithm think it have flame in mistake).

The recall ratio is the ratio of the identified imagees (which really have flame) and all images which really have flame.

According to the forest fire identification algorithm, the authors choose 40 images from video flow randomly to test the identification of flame core color. The results are as follows:

\begin{tabular}{|c|c|c|c|c|}
\hline Category & A & B & C & D \\
\hline Image quantity & 26 & 10 & 2 & 2 \\
\hline
\end{tabular}

Tab.1: test result

A represents that the flame of images are identified by the algorithm;

$B$ represents that the flame of images are not identified by the algorithm;

$\mathrm{C}$ represents that the images which have not flame are identified as the images which have not flame; flames.

D represents that images really don't have any

According to the definitions of the recall ratio and the precision ratio, we can get results as follows: the precision ratio $=\mathrm{A} /(\mathrm{A}+\mathrm{C})=26 /(26+2)$ $=92.86 \%$ $=72.22 \%$

the recall ratio $=\mathrm{A} /(\mathrm{A}+\mathrm{B})=26 /(26+10)$

According to the above data, we can know that the algorithm has the precision ratio and the recall ratio. But in actual operation, the similarity of color feature in video flow has a great influence on the fire identification.

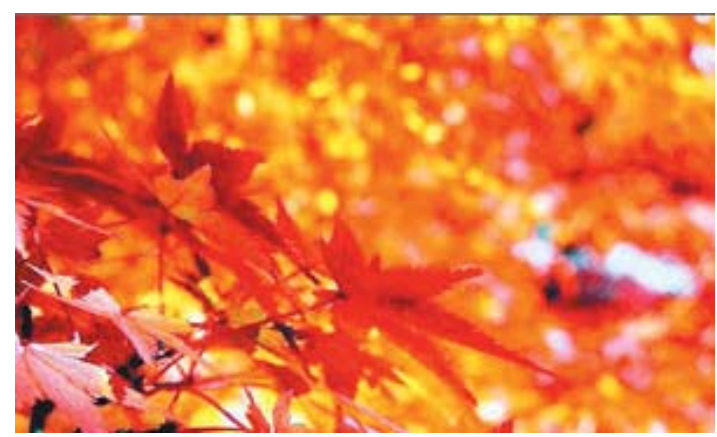

Fig.2: the maple leaf image

For example, in figure 2, according to the human's knowledge structure, we can judge by naked eyes that this is a scene of maple leaves swaying in the wind in deep autumn. But identified by the forest fire identification algorithm, the result is figure 3 :

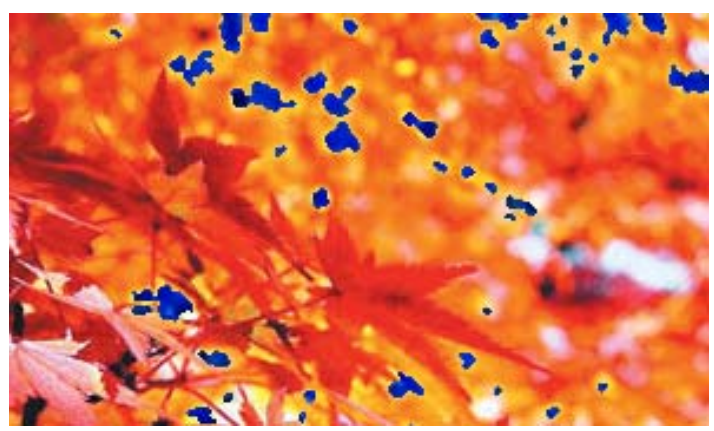

Fig.3: the fire identification of maple leaves picture

Obviously, it is a mistake, and it belongs to situation C. The reason is that the algorithm hasn't considered about the spatial relationship of image.

\section{Conclusions}

According to the features of forest fire remote video surveillance system, the paper puts forward and realizes the algorithm of forest fire identification based on visible light. The algorithm can identify the fire information in the forest fire prevention remote video automatically, and it also has high the recall ratio and the precision ratio. The characteristics of the algorithm are as follows: 
(1) This algorithm breaks the traditional limitation which is based on infrared ray, and can be used in monitoring broader area. It also can analyze the video information directly, extract some features of images and then make use of these features to establish index to search.

(2) The algorithm of forest fire identification based on visible light is an approximate matching algorithm.

The threshold used in the algorithm is based on knowledge, and it mainly comes from professional experience. The features of forest fire are obtained by statistics. However, these datum are great different from the comprehension of people because of the influence of observer's psychology. The content of images has "fuzzy" feature, and images can delivery some un-told informations. On the other hand, people's comprehension about the content of images does not only rely on the statistics but also people's existing knowledge. Therefore, when describing the content of images in index images, it is not enough to adopt some low and simple character vectors such as color, texture, shape, spatial relationship and so on.

\section{Acknowledgement}

This work is partially supported by the National Natural Science Foundation of China (No.60674072), the Natural Science Foundation of Zhejiang, China(Grant No. Y105620) and Science and Technology Foundation of Zhejiang, China(Grant No. 2005C23061, Grant No. 2006C12109)。

\section{References}

[1] Q. Huag, H. Xie, The System of Automatic Monitor for Forest Fire Prevention. Journal of Sichuan University (Natural Science Edition), Sup:87-90, 2001.

[2] D. Zhang, The Application of Topological Forecasting Method of Grey Theory on the Prediction of Forest Fire Disaster. Journal of Fujian College of Forestry, 1: 1-4, 2005.

[3] Y. Shan, Y. Fan, Q. Pang, A Difference-Based Detail-Preserving Adaptive Filter Algorithm. Computer Engineering \& Science, 7: 60-61, 2004.

[4] J. Wang, Modification and application of median filtering algorithm for color image filter.

Journal of Harbin University of Commerce(Natural Sciences Edition), 4:67-68., 2006.

[5] J. Mu, Improving Middle-filter of Erasing Image's Noise. Modern Electronics Technique, 15: 21-24, 2004.
[6] Q. Li, Content based Image Retrival. Journal of Anshan Normal University, 4: 44-45,2005.

[7] R. Duan, Q. Li, Y. Li, Summary of image edge detection. Optical Technique, 3: 415-417, 2005.

[8] H. Yin, H. Fan, A Modified Gradient Algorithm Based on Color Image. Electronic Engineer, 5:41-43, 2005.

[9] H. Dong, X. Chen, W. Fan, Application and Comparison between Different Image Fire Detectors. Optical Technology, 5:51-58,1997.

[10] Y. Li, Y. Fan, C. Hao, A Extremum Method of Image Denoise Based on Image Middle Value. Journal of Computer Applications, 24 (S1):147-148,2004.

[11] J. Wang, Modification and application of median filtering algorithm for color image filter. Journal of Harbin University of Commerce (Natural Sciences Edition), 4: 67-68, 2006.

[12] J. Mu, Improving Middle - filter of Erasing Image's Noise. Modern Electronics Technique, 15: 21-24,2004.

[13] M. Shimoda, A. Sugano, T. Kimura and Y. Watanabe, et.al., Prediction Method of Unburnt Carbon for Utility Boiler Using Image Processing Technique of Combustion Flame. IEEE Transaction of Energy Conversion, 5:640645, 1990. 